REVIEW ARTICLE

\title{
Cultural Awareness: Coining and Cupping
}

\section{Susan Ann Vitale ${ }^{1 *}$ and Thaleshravi Prashad ${ }^{2}$}

\author{
${ }^{1}$ School of Nursing, Molloy College, New York, USA
}

${ }^{2}$ Graduate Nurse Practitioner Student, Molloy College, New York, USA

*Corresponding author: Susan Ann Vitale, PhD, RN, PNP, ANP-C, Professor, School of Nursing, Molloy College, Rockville Center, New York, USA, E-mail: svitale@molloy.edu

The theory of culture care diversity and universality, as described by the renowned anthropologist and nursing leader, Madeleine Leninger [1] suggests that care should be both culturally competent and sensitive to its diverse populations. Within this theory, emphasis is placed on delivering respectful care for patients and their families. The religion and customs of a patient's culture becomes an integral consideration when planning nursing care. Trust and communication are thought to be enhanced within an understanding of the sociocultural and multidimensional aspects of a patient's beliefs. The potential for misconceptions is thereby diminished [2].

The acquisition of cultural competence is an ongoing process that requires self-awareness in a provider. Optimal care also incorporates a sincere interest in various cultures, values and beliefs. This in turn is evidenced by

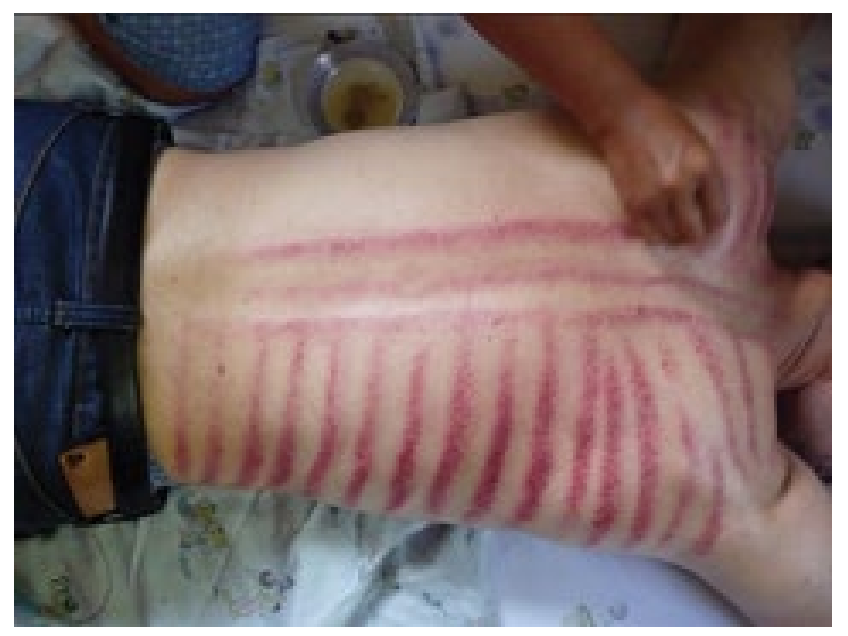

Figure 1: Cao gio (coining). actively listening to patients' expressions of their principles and convictions. Awareness of a patient's culture will then enable a provider to formulate individualized care plans with respect, genuine concern and attention to cultural details $[3,4]$.

The cultural beliefs of providers and those of patients or their families may differ widely or present ethical dilemmas. There may not always be clear solutions to issues that cannot be easily reconciled [5]. As an example of misconceptions by nursing staff is illustrated through a case scenario (See Clinical Scenario) in which multiple body marks on an elderly man resembling burns and bruises caused concern for his nurses. Elder abuse was suspected until it was found that the skin marks in question were actually a result of a cultural practice known as Cao gio, coining or press scraping.

\section{The Practice of Cao Gio (Coining)}

Gua sha [6] or coining translates to "scraping or bruising" (Figure 1 and Figure 2). It has also been termed Cao gio which means "catch the wind". It is an important part of medicine in various cultures including southeastern Asian. Skin lubricated with an oil is scraped with a ceramic spoon, worn coin or metal cap. The elongated bruises resulting from this procedure are considered signs of balance, stimulated blood flow and the restoration of health. These measures are taken to correct imbalances attributed to poor nutrition, stress, fatigue, overexposures to "wind", and asymmetries between yin and yang. The balance of Yang and Yang from Chinese philosophy indicates negative and positive interacting forces that maintain the harmony of the universe [7]. Success in ridding the body of bad "wind"

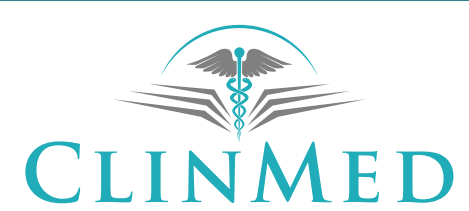

INTERNATIONAL LIBRARY
Citation: Vitale SA, Prashad T (2017) Cultural Awareness: Coining and Cupping. Int Arch Nurs Health Care 3:080. doi.org/10.23937/2469-5823/1510080

Received: July 27, 2017: Accepted: September 18, 2017: Published: September 20, 2017

Copyright: (c) 2017 Vitale SA, et al. This is an open-access article distributed under the terms of the Creative Commons Attribution License, which permits unrestricted use, distribution, and reproduction in any medium, provided the original author and source are credited. 


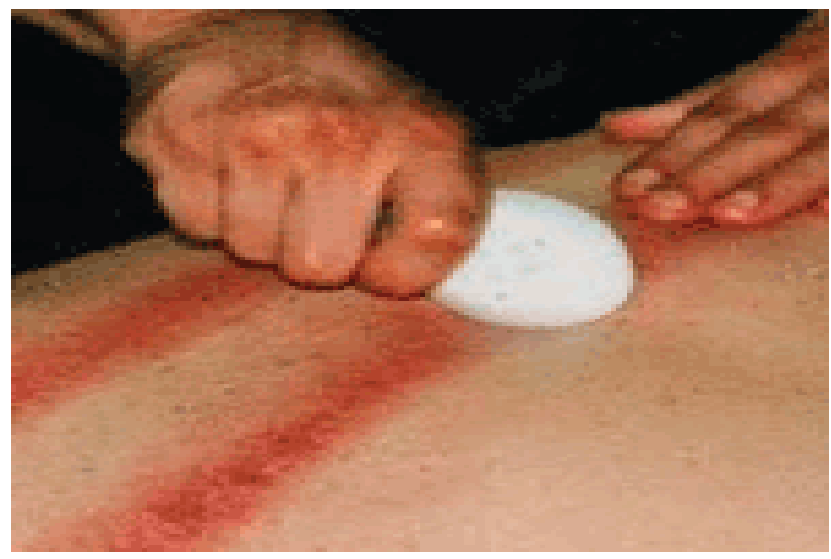

Figure 2: Gua sha (ceramic spoon scrapping).

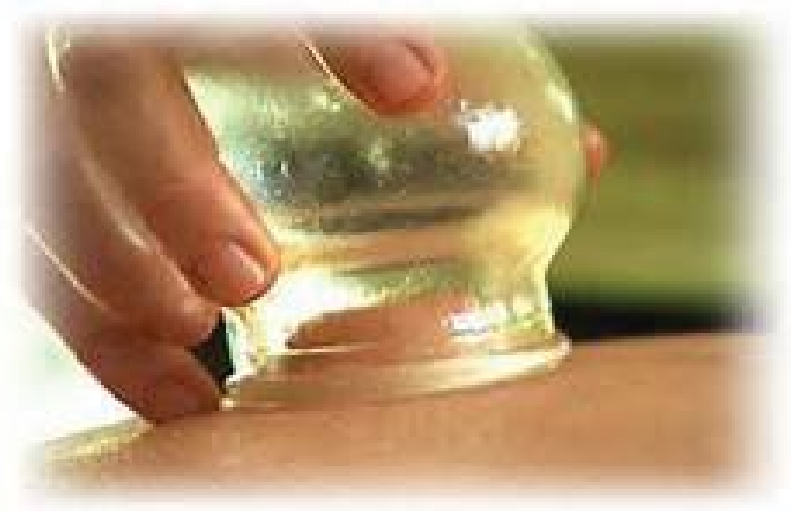

Figure 3: Dry cupping.

is estimated by the degree of redness evident after the scraping treatment. The shade of the marks illustrates the severity of the illness present [8]. The skin is vigorously rubbed in a linear manner for 15-20 minutes over the spine, ribs or head. The rubbing is continued until redness occurs. Coining is used to treat many symptoms such as cough, cold, fever or headache. Heated ointments are rubbed into the skin and may contain herbs, camphor, menthol, wintergreen, eucalyptus, peppermint, or cinnamon oil. The minor burns, abrasions, or bruising that subsequently develop will take a few days to resolve. They can be misinterpreted as abuse [9]. Coining may also be used with children and infants. A hot coin may be placed on the child's skin to stop uncontrollable crying. Coining may also be combined with the practice of cupping. Both procedures have been found to be used in children. In the United States, this may be considered maltreatment [10].

\section{The Practice of Dry and Wet Cupping}

Cupping is another type of ancient therapy traced back to $3000 \mathrm{BC}$. Renewed interest in this treatment has resulted in its use by a variety of current practitioners. Massage and physical therapists may utilize cupping in the treatment of pain. Circular suction cups made of glass, bamboo, or earthenware are applied to dry skin (Figure 3). The suction causes a bruise or hematoma. Wet cupping involves small skin incisions made in the

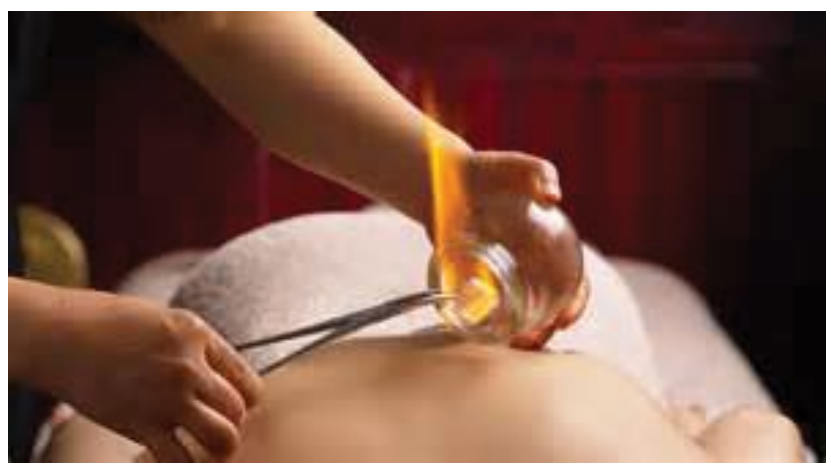

Figure 4: Warmed air to create suction.

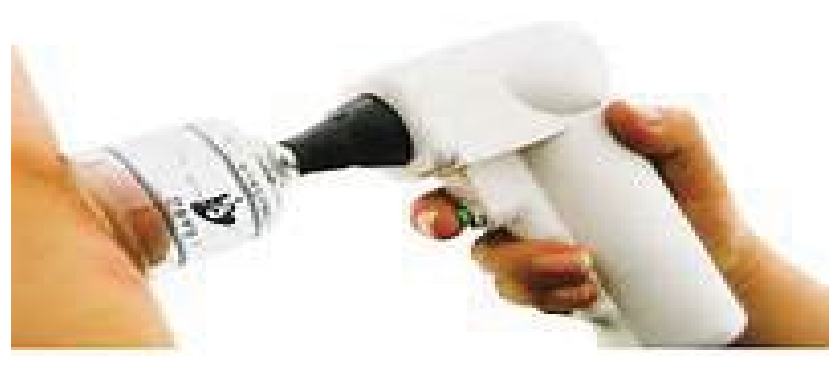

Figure 5: Suction by pump.

suction area [11]. Such skin markings may also be misconstrued for maltreatment or abuse.

There are a number of methods available with which to create the suction. A traditional technique involves warming the air inside a cup by means of a fire started with alcohol, herbs or paper. When the fire dies out, the cup is placed upside down on the patient's skin for about 5-10 minutes. As the air inside the cup cools, the vacuum then created causes blood vessels to expand (Figure 4).

A more modern version creates the vacuum with a rubber pump (Figure 5). Another method simulates a massage with silicone cups moved across the skin. For wet cupping, mild 3 minute suction is created. This is followed by superficial skin incisions and then a second suction to draw out small amounts of blood so as to remove toxins or harmful substances. Antibiotic ointment and bandages may be placed over the incisions that heal within about 10 days. Side effects may include a range of discomforts, burns, bruises and infections [12].

Cupping has also been used to treat chronic low back pain [13]. Research studies have been conducted on a variety of additional indications. Acne, cervical spondylosis, cancer pain, trigeminal neuralgia, brachialgia, carpal tunnel syndrome and herpes zoster have all been treated with cupping. Future research should determine its efficacy, compared to standard treatment and pharmacological relief $[12,14]$.

\section{Conclusion}

Patient care should always be culturally sensitive. Inaccurate assumptions may be made by health care 
providers about patients who speak a different language. Health care providers need to develop transcultural skills so as to care for individuals of multicultural backgrounds. Opportunities to enhance such awareness can be provided with in training and nursing school programs or as in-service educational programs for working professionals. They must identify the cultural preferences of their patients. Providers can prepare for possible cultural conflicts even though not all can be reasonably predicted. As providers, we should also strive for awareness of our own biases and backgrounds while widening and strengthening our foundation of knowledge so as to effectively practice within the ethnic mosaics we may encounter.

\section{Clinical Scenario}

An 83-year-old Vietnamese man was admitted to an acute care setting for hepatic encephalopathy and altered mental status. He presented in the emergency department at 1900 hours from home and was accompanied by his wife. They only spoke Vietnamese and did not understand much English. An assessment of the patient's skin noted multiple elongated ecchymotic markings along his back and ribs. The staff immediately became concerned about the possibility of elder abuse. One hour later he was admitted for observation and treatment. A full assessment and examination was performed. The family arrived and advised the admitting nurse that the marks were from Cao gio, commonly termed coining. The patient's wife performed the procedure prior to coming to the hospital. According to the patient's son, warm oil was first used in a massage to induce a mental state of stillness or a sense of calm. A coin was then repetitively rubbed against the skin moving away from the heart. Although the resulting abrasions can be misconstrued as abuse, this practice is believed to enhance good health in stabilizing imbalances between yin and yang. The staff was then able to treat this patient effectively while respecting the health practice of coining. The nurse learned through an interpreter that this patient's family had been practicing Cao gio for decades. They had also been receiving this therapy from a healer. While the marks were not medically serious, the concerns that it engendered were alarming to an uninformed staff.

\section{References}

1. Ray MA (2012) Remembering Madeleine M Leininger, 1925-2012. International Journal for Human Caring 16: 6-8.

2. McFarland M, Eipperle M (2008) Culture care theory: A proposed practice theory guide for nurse practitioners in primary care settings. Contemp Nurse 28: 48-63.

3. Maier-Lorentz MM (2008) Transcultural nursing: Its importance in nursing practice. J Cult Divers 15: 37-43.

4. Nelson J (2006) Madeleine Leininger's culture care theory: The theory of culture care diversity and universality. International Journal for Human Caring 10: 50-56.

5. Howe E (2016) Respecting other cultures. Military Medicine 181: $102-103$.

6. Liu M, Chen K (2013) Gua Sha, an ancient technical management, for certain illness. Chin J Integr Med 19: 3-4.

7. Yin and yang Collins English Dictionary - Complete \& Unabridged $\left(10^{\text {th }}\right.$ edn $)$.

8. Zamani R (2010) Coining: What you need to know. Fact Sheets for Families.

9. (2016) Child Abuse: What is Coining? Stanford Medicine.

10. Berg J, Morphew T, Tran J, Kilgore D, Galant SP (2016) Prevalence of complementary and alternative medicine usage in Vietnamese American Asthmatic Children. Clin Pediatr (Phila) 55: 157-164.

11. Rozenfeld E, Kalichman $L$ (2016) New is the well-forgotten old: The use of dry cupping in musculoskeletal medicine. J Bodyw Mov Ther 20: 173-178.

12. Web MD (2016) Cupping therapy. 1-2.

13. Markowski A, Sanford S, Pikowski J, Fauvell D, Cimino D, et al. (2014) A Pilot study analyzing the effects of chinese cupping as an adjunct treatment for patients with subacute low back pain on relieving pain, improving range of motion, and improving function. J Altern Complement Med 20: 113117.

14. Jong-In K, Myeong Soo L, Dong-Hyo L, Boddy K, Ernst E (2011) Cupping for treating pain: A systematic review. Evid Based Complement Alternat Med 2011: 467014. 